\title{
Determinación de coeficientes genéticos en tres cultivares y un híbrido de maíz ${ }^{1}$
}

\author{
Marilis Esteves ${ }^{2}$,Elvin Román-Paoli ${ }^{3}$, James S. Beaver ${ }^{3}$, \\ Miguel A. Muñoz $z^{3}$ y Arístides Armstrong ${ }^{3}$
}

J. Agric. Univ. P.R. 96(1-2):57-75 (2012)

\begin{abstract}
RESUMEN
Se realizó un estudio en la Subestación Experimental Agrícola de Lajas de la Universidad de Puerto Rico para determinar el comportamiento agronómico de cuatro genotipos de maíz. El objetivo de esta investigación fue determinar los coeficientes genéticos de tres cultivares locales de maíz: 'Mayorbela' (M), 'Diente de Caballo' (DC), 'Chulo' (Ch) y de un híbrido transgénico de Pioneer Hi-Bred International, Inc. (híbrido Pioneer) para utilizarlos en el modelo CERES-Maize para predecir el comportamiento de estos genotipos bajo condiciones tropicales. Se determinaron los coeficientes genéticos para el crecimiento y desarrollo del maíz, los cuales incluyeron: duración de la fase juvenil del cultivo (P1), duración de la fase de acumulación de materia seca en el grano (P5), número potencial de granos por mazorca (G2), y crecimiento diario lineal del grano (G3). El crecimiento del cultivo se expresó utilizando el concepto de unidades termales (Td). Además, se estimaron otras variables de crecimiento tales como índice de área foliar y biomasa total acumulada. Se establecieron dos experimentos en el campo, en febrero 2004 y en marzo 2005. Los estimados de P1 durante el experimento del 2004 fueron 219 Td para el híbrido de Pioneer, y 253, 252 y 362 Td para M, DC y Ch, respectivamente. En el segundo experimento, realizado en el 2005, los valores estimados de P1 resultaron mayores a los obtenidos en el 2004; siendo de 352, 422, 479 y 405 Td para el híbrido Pioneer, M, DC y Ch, respectivamente. En el 2004 los valores de P5 fueron los esperados para todos los genotipos excepto para Ch. Estos valores corresponden a $715 \mathrm{Td}$ para el híbrido Pioneer; $757 \mathrm{Td}, 691 \mathrm{Td}$, y $413 \mathrm{Td}$, para M, DC y Ch, respectivamente. En el 2005, los estimados de P5 fueron mayores que en el 2004, especialmente para Ch el cual casi se duplicó. En el 2004, el híbrido Pioneer obtuvo el mayor valor de G2, correspondiente a 616 granos por mazorca. No se detectaron diferencias significativas para G2 entre los genotipos estudiados; los valores de G2 variaron entre 529 y 616 granos por mazorca. En el caso de G3 los estimados fueron: $7.7,6.7,5.7$ y 3.7 $\mathrm{mg} / \mathrm{di}$ a para el híbrido Pioneer, M, DC y Ch, respectivamente. Mientras, los valores estimados para G3 en el 2005 fueron $8.5,6.9,9.6$ y $7.1 \mathrm{mg} / \mathrm{día}$ para el híbrido Pioneer, $\mathrm{M}, \mathrm{DC}$ y $\mathrm{Ch}$, respectivamente. No se encontraron diferencias significativas entre genotipos para índice de área foliar ni para biomasa
\end{abstract}

${ }^{1}$ Manuscrito sometido a la Junta Editorial el 12 de enero de 2012.

${ }^{2}$ Ex estudiante graduado, Departamento de Agronomía y Suelos, Univ. de Puerto Rico-Recinto Universitario de Mayagüez.

${ }^{3}$ Catedráticos, Departamento de Cultivos y Ciencias Agroambientales, Colegio de Ciencias Agrícolas, Universidad de Puerto Rico-Recinto Universitario de Mayagüez. HC02 Box 11656, Lajas, PR 00667-9714. autor para correspondencia: elvin.roman@upr.edu 
acumulada. El rendimiento más alto en el 2004 lo obtuvo M con 4,148 kg/ ha, seguido muy de cerca por el híbrido Pioneer, siendo esta diferencia no significativa. En el $\mathbf{2 0 0 5}$ el híbrido Pioneer superó significativamente a M por $14 \%$.

Palabras clave: coeficientes genéticos, maíz, CERES-Maize, rendimiento

\author{
ABSTRACT \\ Genetic coefficient determination for three maize cultivars and one \\ hybrid
}

A study was conducted at the Lajas Substation of the University of Puerto Rico to determine the agronomic performance of four maize genotypes. The objective of this research was to determine the genetic coefficients of three maize cultivars, 'Mayorbela' (M), 'Diente de Caballo' (DC), 'Chulo' (Ch), and a transgenic hybrid from Pioneer Hi-Bred International, Inc. (Pioneer hybrid) to be utilized by the CERES-Maize model to predict performance under tropical conditions. The experiments determined genetic coefficients for maize growth and development including duration of the juvenile phase of the plant (P1); duration of biomass accumulation in the grain (P5); potential kernel number per cob (G2), and kernel linear growth rate (G3). Crop growth was expressed using the concept of thermal units (Td). Also determined were leaf area index and total biomass accumulation. Field experiments were planted February 2004 and March 2005. Estimates of PI from the experiment planted in 2004 were 219 Td for Pioneer hybrid, 253 Td for M, 252 Td for DC, and $362 \mathrm{Td}$ for Ch. In 2004 the P5 values were 715, 757, 691, and 413 Td for Pioneer hybrid, M, DC, and Ch, respectively. Nevertheless, the Pioneer hybrid produced the highest G2 value, 616 kernels per cob. There were no significant differences in G2 among genotypes, which varied from 529 to 616 kernels per cob. The G3 values were 7.7, 6.7, 5.7 and $3.7 \mathrm{mg} / \mathrm{day}$ for Pioneer hybrid, M, DC and Ch, respectively. There were no significant differences among genotypes for leaf area index and biomass accumulation. Estimates of P1 from the 2005 experiment were higher than those obtained on 2004. The P1 values from the experiment planted in 2005 were $352 \mathrm{Td}$ for Pioneer hybrid, $422 \mathrm{Td}, 479 \mathrm{Td}$, and $405 \mathrm{Td}$ for $\mathrm{M}, \mathrm{DC}$ and $\mathrm{Ch}$, respectively. The P5 values were $756 \mathrm{Td}$ for Pioneer hybrid; $755 \mathrm{Td}, \mathrm{M} ; 795 \mathrm{Td}, \mathrm{DC}$; and $777 \mathrm{Td}$, Ch. The estimated values for G3 were 8.5, 6.9, 9.6, and $7.1 \mathrm{mg} /$ day for Pioneer hybrid, M, DC, and Ch, respectively. No significant differences were found among genotypes for biomass accumulation. In 2004 'Mayorbela' produced the greatest seed yield $(4,148 \mathrm{~kg} / \mathrm{ha})$ followed closely by Pioneer with no significant difference, whereas in 2005 Pioneer hybrid surpassed 'Mayorbela' by $14 \%$.

Key words: genetic coefficients, maize, CERES-Maize, yield

\title{
INTRODUCCIÓN
}

Los modelos de simulación son representaciones matemáticas de procesos que podemos observar y/o medir. Estos modelos están basados en procesos físicos, químicos y biológicos de las plantas y del ambiente (Freebaim et al., 1993). La solución de estas representaciones 
matemáticas utilizando un programa de computadora se conoce como simulación.

El utilizar un modelo de simulación nos permite estudiar algunos aspectos de la producción agrícola en menos tiempo y analizar diferentes variables simultáneamente, lo cual no puede hacerse eficientemente con experimentos tradicionales (Román-Paoli et al., 2000). Muchos modelos de simulación utilizan el concepto de coeficientes genéticos (CG) para caracterizar diferentes genotipos. Los CG son parámetros que describen la interacción genotipo x ambiente (IBSNAT, 1993). Para determinar los CG de un cultivo, se utilizan dos métodos: (1) determinación experimental directa en experimentos establecidos en el campo para esos propósitos (Ogoshi et al., 1989), y (2) utilización de procesos computarizados manejando datos históricos de variables fenológicas (Hunt et al., 1993; Román-Paoli et al., 2000).

El modelo CERES-Maize y la serie CROPGRO (para simular leguminosas) son ejemplos de algunos de los modelos que utilizan el concepto de CG. El modelo CERES-Maize simula crecimiento, fenología y rendimiento, utilizando datos del suelo, clima e insumos de manejo (Carberry et al., 1989; Román-Paoli et al., 2000). CERES-Maize simula aspectos fisiológicos de crecimiento (Jones y Kiniry, 1986). Ambos modelos son parte de un grupo de modelos incluidos en el programa DSSAT (Decision Support Systems for Agrotechnology Transfer) que es administrado por el grupo internacional ICASA (International Consortium for Agricultural Systems Applications) (DSSAT, 1998). Los modelos incluidos en DSSAT utilizan características hereditarias, crecimiento de hojas, tallos y raíces, acumulación y repartición de biomasa, balance hídrico en el suelo e influencia del déficit hídrico sobre el crecimiento y desarrollo del cultivo. Otros factores considerados son el balance en el suelo e influencia del déficit de nitrógeno sobre el crecimiento y desarrollo del cultivo. El balance de otros macronutrimentos (fósforo y el potasio), al igual que el control de plagas y enfermedades, hasta el momento no son considerados factores limitantes en el programa DSSAT (Jones y Kiniry, 1986). Actualmente existen rutinas computarizadas que están siendo evaluadas para simular el efecto de otros nutrimentos además del nitrógeno y el efecto de algunas plagas y enfermedades (Tsuji et al., 1998).

En estos modelos, el crecimiento de los cultivos se expresa utilizando el concepto de unidades termales (Td). Con este concepto se presume que el crecimiento expresado en tiempo termal depende únicamente de la temperatura y que la respuesta en crecimiento del cultivo es lineal. Basado en este concepto, el cultivo crece o se desarrolla cuando la temperatura promedio ambiental es mayor a la temperatura base $(\mathrm{Tb})$ y menor a una temperatura máxima. En el caso de maíz, Tb es igual a $8^{\circ}$ 
C, la temperatura óptima para el llenado del grano de maíz es de $30^{\circ} \mathrm{C}$, y la temperatura máxima es $34^{\circ} \mathrm{C}$ (Carberry et al., 1989). Con el modelo, $\mathrm{Td}$ se calcula diariamente utilizando las temperaturas diarias de la localidad que está siendo estudiada aplicando la siguiente ecuación [1].

$$
T d=\sum_{i=0}^{n} \frac{T m a x+\operatorname{Tmin}}{2}-T b
$$

Donde:

$\mathrm{Td}=$ unidades termales

Tmax $=$ temperatura máxima diaria, ${ }^{\circ} \mathrm{C}$

Tmin = temperatura mínima diaria, ${ }^{\circ} \mathrm{C}$

$\mathrm{Tb}=$ temperatura base, ${ }^{\circ} \mathrm{C}$

$\mathbf{n}=$ número de días en el periodo

Otro aspecto importante que el modelo tiene que considerar es la fenología del cultivo. En maíz las etapas fenológicas se dividen en etapas de crecimiento vegetativo y crecimiento reproductivo. El crecimiento vegetativo se expresa en términos del número de hojas completamente desarrolladas. Una hoja de maíz se considera completamente desarrollada cuando expone en su totalidad la lámina y la yagua. Una planta de maíz se encuentra en la etapa vegetativa $V_{1}$ cuando tiene expuesta en su totalidad la primera hoja y así sucesivamente, $V_{2}, V_{3}$,..hasta $V_{(n)}$, la cual representa la última hoja completamente desarrollada antes de aparecer la inflorescencia masculina (Ritchie et al., 1992). Normalmente el maíz puede desarrollar de 21 a 30 hojas. Cuando germina la semilla la primera hoja expuesta es la cotiledonaria, designándose como VE.

En los modelos de cereales tales como CERES-Maize, los CG son designados con una letra seguida por un número. En el Cuadro 1 se describen los CG designados para maíz en el modelo. Se designa como P1 las unidades termales (Td) que transcurren desde que la semilla germina (emerge del suelo) hasta que el cultivo completa la etapa juvenil, lo cual ocurre cuatro días antes de la diferenciación del meristemo o yema apical (cuando cambia de etapa vegetativa a reproductiva). El periodo durante el cual la planta es sensible a cambios en el fotoperiodo se designa como P2. Los valores de P2 varían desde cero hasta uno (Tsuji et al., 1994). Un valor de uno significa que la planta demora un día adicional en diferenciar su yema apical por cada hora de luz en exceso de $12 \mathrm{~h}$. En Puerto Rico, el día más largo del año es el 21 de junio con un fotoperíodo de 13.13 horas. Algunos cultivares de maíz son sensitivos al fotoperiodo; sin embargo, la mayoría de los cultivares comerciales de maíz son insensitivos al fotoperiodo. De acuerdo a 
CUaDro 1.-Definición de los coeficientes genéticos utilizados en CERES-Maize.

\begin{tabular}{ll}
\hline Coeficiente & \multicolumn{1}{c}{ Descripción } \\
PHINT & $\begin{array}{l}\text { "Phylochron interval"; intervalos, en unidades termales (Td), entre la apa- } \\
\text { rición de cada hoja sucesiva }\end{array}$ \\
P1 & $\begin{array}{l}\text { Período, en Td, desde emergencia de la semilla hasta el final de la etapa } \\
\text { juvenil durante el cual la planta no responde a cambios en fotoperíodo }\end{array}$ \\
P2 & $\begin{array}{l}\text { Período, en Td, durante la etapa juvenil en el cual la planta es sensible a } \\
\text { cambios en fotoperíodo }\end{array}$ \\
P3 & $\begin{array}{l}\text { Período, en Td, desde iniciación de la inflorescencia masculina hasta el final } \\
\text { del crecimiento de hojas y la formación de la inflorescencia femenina }\end{array}$ \\
P4 & $\begin{array}{l}\text { Período, en Td, entre la fecha de florecida hasta el comienzo del llenado del } \\
\text { grano }\end{array}$ \\
P5 & $\begin{array}{l}\text { Período, en Td, de llenado del grano desde fecha de florecida femenina has- } \\
\text { ta fecha de madurez fisiológica }\end{array}$ \\
P6 & $\begin{array}{l}\text { Período, en Td, desde el final de llenado del grano hasta fecha de madurez } \\
\text { fisiológica }\end{array}$ \\
P7 & $\begin{array}{l}\text { Presiembra } \\
\text { P8 }\end{array}$ \\
Período entre germinación y emergencia de la semilla \\
G2 & $\begin{array}{l}\text { Período, en Td, desde germinación a emergencia de la semilla } \\
\text { Número potencial de granos por mazorca por planta }\end{array}$ \\
G3 & $\begin{array}{l}\text { Velocidad de llenado del grano durante la etapa lineal bajo óptimas condi- } \\
\text { ciones (mg/día) }\end{array}$ \\
\hline
\end{tabular}

Td - Unidades termales, las cuales son calculadas utilizando la ecuación [1] antes mencionada y los datos climatológicos de la localidad en estudio.

Román-Paoli et al. (2000) el modelo CERES-Maize es insensible al P2, por lo que no se requiere de un estimado preciso para P2.

El P5 es el CG que describe la etapa de crecimiento que comprende el período desde la aparición de la inflorescencia femenina hasta la etapa de madurez fisiológica del grano. La madurez fisiológica se define como la etapa de crecimiento y desarrollo en donde se determina el peso final de la semilla. En el caso de CG para etapas reproductivas, el modelo CERES-Maize utiliza los parámetros G2 y G3 (Ritchie et al., 1986). El número potencial de semillas (G2) es un estimado del número potencial de granos que produce una mazorca que está creciendo sin limitaciones (Ritchie et al., 1986). El coeficiente G3 describe la acumulación diaria de materia seca en la fase lineal de crecimiento de la semilla. Un aumento o disminución de G3 tiene un gran efecto en el rendimiento y el peso de la semilla, por lo cual el modelo es bien sensitivo al coeficiente G3. Existen tres fases para el crecimiento de la semilla: la fase juvenil del grano, cuando está en una etapa lechosa y acumula materia seca muy lentamente; la fase lineal, cuando el grano acumula materia seca rápidamente; y la fase decreciente, hasta que ocurre la formación de una línea negra dentro de la semilla, evidencia 
de que la misma ha alcanzado su madurez fisiológica. Los demás coeficientes mencionados en el Cuadro 1 no varían mucho entre genotipos, por lo que fueron incluidos en CERES-Maize como parámetros.

Entre los cultivares de maíz tradicionales de polinización abierta en Puerto Rico se encuentran 'Mayorbela', 'Chulo' y 'Diente de Caballo'; cultivares que fueron mejorados genéticamente por la Estación Experimental de Agricultura Tropical (TARS, por sus siglas en inglés) del Departamento de Agricultura Federal. Estos cultivares han sido evaluados bajo diferentes condiciones de manejo y suelos, con buenos rendimientos (Sotomayor-Ríos et al., 1980).

'Mayorbela' posteriormente fue seleccionado para mejores características y fue liberado nuevamente como 'Mayorbela 05' (Beaver et al., 2006). Este cultivar se recomienda tanto para producción de grano seco y mazorcas verdes como para ensilaje. Bajo condiciones ambientales favorables 'Mayorbela' puede producir hasta $7,000 \mathrm{~kg} / \mathrm{ha}$ de grano seco. La mazorca es de tamaño mediano y puede producir de 27,000 hasta 30,000 mazorcas por hectárea para una cosecha de maíz fresca. El maíz 'Chulo'es una población mejorada que tiene el grano cristalino pequeño de color amarillo pálido y también se recomienda para la alimentación de gallos de pelea (Meneses-Ojeda, 1992). 'Diente de Caballo' tiene un grano dentado, la planta es medianamente alta y puede producir hasta dos mazorcas (López, 1994).

El objetivo principal de este estudio fue determinar los coeficientes genéticos de tres cultivares de polinización abierta adaptados a las condiciones tropicales, y de un híbrido de Pioneer (Pioneer Hi-Bred International, Inc.). ${ }^{4}$ Estos coeficientes genéticos se utilizarán en el modelo CERES-Maize para simular producción de maíz a gran escala en las reservas agrícolas de Puerto Rico.

\section{MATERIALES Y MÉTODOS}

\section{Localidad}

La investigación se realizó en la Subestación Experimental Agrícola de Lajas de la Universidad de Puerto Rico, Recinto Universitario de Mayagüez. El clima de la zona es seco, con una precipitación que varía entre 762 y $889 \mathrm{~mm} /$ año (Lugo López, 1995). Los suelos son fértiles, profundos, con un alto contenido de arcillas expandibles 2:1 (vermicu-

\footnotetext{
${ }^{4}$ Los nombres de compañías y de marcas registradas sólo se utilizan para proveer información específica y su uso no constituye garantía por parte de la Estación Experimental Agrícola de la Universidad de Puerto Rico, ni endoso sobre otros productos o equipo que no se mencionan.
} 
lita: montmorillonita) (Carte, 1965; Gierbolini, 1975). El suelo donde se estableció el experimento pertenece a la serie Fraternidad (Typic Haplustert). La preparación del suelo consistió de una aradura y un rastrillado.

\section{Manejo del área experimental}

Se establecieron dos siembras experimentales de maíz; la primera siembra se estableció el 19 de febrero de 2004. La distancia entre las plantas dentro de las hileras fue de $0.28 \mathrm{~m}$ y $0.91 \mathrm{~m}$ entre hileras para una densidad de siembra de 3.6 plantas por metro cuadrado. Para estimar CG bajo condiciones de campo se requiere que la planta exprese su potencial, por lo que es necesario sembrar a baja densidad para eliminar o reducir el estrés hídrico y la competencia por plagas y enfermedades. Para el control de malezas se aplicó el herbicida pendimetalina como pre-emergente a razón de $0.817 \mathrm{~g} i \mathrm{a} / \mathrm{m}^{2}$. Se utilizó riego por goteo para lo cual se instaló un hidrante y laterales con emisores espaciados a $30 \mathrm{~cm}$ conectados a una manga de polietileno de $5 \mathrm{~cm}$ de diámetro. El agua de riego se aplicó a una presión aproximada de 1.74 $\mathrm{kPa}$. El control de insectos se realizó mayormente con aplicaciones de insecticidas de contacto como methomyl y aplicaciones de insecticidas biológicos con las esporas de Bt.

Se aplicó fertilizante a razón de $225-113-225 \mathrm{~kg} / \mathrm{ha}$ para N-P-K, utilizando la formulación 10-10-08 granulado postsiembra dividido en dos aplicaciones. La primera aplicación de fertilizante granulado se realizó dos semanas después de la siembra, utilizando el $40 \%$ de la dosis. El restante $60 \%$ del fertilizante se aplicó en cuatro fertigaciones a intervalos de dos semanas después de la primera aplicación, hasta los 55 días después de la siembra. Las fuentes solubles de nutrimentos fueron urea, ácido fosfórico y sulfato de potasio para $\mathrm{N}, \mathrm{P}_{2} \mathrm{O}_{5}$ y $\mathrm{K}_{2} \mathrm{O}$, respectivamente. Las fechas de fertigación correspondientes fueron 10 de marzo, 24 de marzo, 7 de abril, y 21 de abril del 2004 .

La segunda siembra se realizó el 2 de marzo de 2005. Se mantuvo la misma densidad de siembra y dosis de fertilizantes que en el 2004, pero esta vez se utilizó la formulación granulada 15-05-10. La primera aplicación, de $40 \%$ de la dosis, se realizó dos semanas después de la germinación del maíz. A diferencia de la siembra del 2004 , la segunda aplicación, del $60 \%$ del fertilizante, se realizó 55 días después de la germinación. En el 2004 se fertigó aún cuando el contenido de humedad en el suelo era alto, lo que posiblemente causó que el fertilizante se perdiera por escorrentía y/o lixiviación. Por lo cual, en el 2005 se aplico el fertilizante en forma granulada. Las demás prácticas de manejo se mantuvieron iguales a las del año 2004. 


\section{Diseño experimental y tratamientos}

Para ambas siembras experimentales (2004 y 2005) se utilizaron cuatro genotipos de maíz: 'Mayorbela', 'Chulo', y 'Diente de Caballo' y un híbrido modificado genéticamente con genes de Bacillus thuringiensis (RX8284G 1769 WCV"AF) producido por Pioneer Hi-Bred International, Inc. El diseño experimental utilizado durante 2004 consistió de bloques completamente aleatorizados, arreglados en parcelas divididas con cuatro repeticiones. Los genotipos de maíz fueron asignados a la parcela principal y las parcelas divididas correspondieron a los muestreos a través del tiempo. En el 2005, con el propósito de aumentar la precisión experimental, se modificó el diseño experimental a uno de bloques completamente aleatorizados arreglados en parcelas divididas con ocho repeticiones. Debido a que la germinación de la semilla de maíz no fue uniforme, el análisis de varianza de los datos obtenidos en el 2005 se realizó utilizando el número de plantas por parcela como covariable. Las medias se separaron utilizando la prueba de diferencia mínima significativa, con un $5 \%$ de probabilidad del error.

Determinación de los coeficientes genéticos P1 y P5

La primera variable medida fue la fecha de germinación (emergencia de la semilla), la cual se determinó cuando $50 \%$ o más de las semillas sembradas en cada parcela habían germinado. Además, se determinó la fecha de la diferenciación de la yema apical. Para determinar esta etapa con precisión se debe disectar la planta de maíz cuando se encuentran en la etapa V6 (con seis hojas completamente desarrolladas). Utilizando un estereoscopio se observa la diferenciación de la yema apical (esto ocurre cuando la yema apical de la planta deja de producir hojas para producir la inflorescencia masculina). El muestreo de dos plantas por parcela se continúa en días alternados hasta observar la diferenciación de la yema apical en el $50 \%$ de las plantas seleccionadas. La fecha de florecida masculina se registra cuando el $50 \%$ de las plantas en el campo presenta la inflorescencia masculina. Entre tres a cuatro días después de la florecida masculina comienza a desarrollarse la inflorescencia femenina. La fecha de florecida femenina se determina al observar que $50 \%$ de las plantas en el campo presentan la formación de la inflorescencia femenina. La fecha de madurez fisiológica se determinó disectando el grano de maíz hasta observarse la formación de la línea negra en el 50\% de las mazorcas muestreadas (Nielsen et al., 2002).

Se determinaron los CG P1 y P5, según definido en el Cuadro 1. Las unidades termales (Td) para estimar P1 y P5 se calculan utilizando los datos climáticos medidos en la estación meteorológica de la subestación de Lajas y la ecuación [1]. El P2 expresa la sensibilidad al fotope- 
ríodo del maíz y normalmente se determina en ambientes controlados; por esta razón no se determinó en este estudio (Kiniry et al., 1983).

Determinación de los coeficientes genéticos G2 y G3

El número potencial de granos por mazorca $(\mathrm{G} 2)$ se determinó cuantificando el número total de granos por mazorca de una muestra de 25 mazorcas por parcela. Estas mazorcas se tomaron de las dos hileras del centro de cada parcela, las cuales se mantuvieron intactas durante el experimento. El estimado de G2 se obtuvo utilizando el promedio del 95\% del valor máximo de granos por mazorca por parcela.

Los muestreos para determinar el crecimiento lineal del grano (G3) comenzaron tan pronto las mazorcas iniciaron su desarrollo. En el 2004, se tomaron dos mazorcas por parcela, mientras que en el 2005 solo se tomó una para un total de ocho mazorcas por tratamiento. A cada mazorca se le tomó 20 semillas del centro que se secaron en el horno a $60^{\circ} \mathrm{C}$ hasta alcanzar peso uniforme. Se tomó su peso seco y se dividió entre el número de granos para determinar el peso promedio por semilla (Duncan y Hatfield, 1964). Este procedimiento se realizó semanalmente hasta que la planta alcanzó la etapa de madurez fisiológica. Se realizó un análisis de regresión lineal, entre la acumulación de materia seca semanal del grano y el tiempo transcurrido hasta la fecha de madurez fisiológica. La pendiente de esta regresión, descartando los datos donde la acumulación no es lineal, es un estimado del coeficiente genético G3 (Tsuji et al., 1994).

Índice de área foliar, acumulación de biomasa y rendimiento

Para medir el área foliar de la planta se utilizó el instrumento $\mathrm{Li}$ 3100 (LI-COR, Lincoln, NE). Se determinó la biomasa total acumulada en la planta, número total de hojas e índice de área foliar (IAF) utilizando dos plantas por parcela en el 2004 y solo una en el 2005. Para estimar biomasa, las plantas se secaron por 94 horas en un horno, a $60^{\circ} \mathrm{C}$, hasta alcanzar un peso uniforme. El rendimiento se determinó cosechando las mazorcas de las dos hileras del centro de cada parcela con un área de $11.5 \mathrm{~m}^{2}$.

\section{RESULTADOS Y DISCUSIÓN}

El maíz 'Chulo' tuvo problemas para germinar, por lo que se resembró el 8 de marzo del 2004. Debido a la diferencia en la fecha de siembra, las etapas del 'Chulo' no se desarrollaron a la par con las de los demás genotipos, por lo que las variables se analizaron independientemente de los demás genotipos aunque están presentadas en conjunto. En el 2005, el maíz 'Diente de Caballo' tuvo un porcentaje bajo de germinación. Debido al bajo número de plantas presentes, no se consi- 
deraron las variables de rendimiento, área foliar, biomasa acumulada ni el coeficiente G2.

\section{Coeficientes genéticos P1 y P5}

Para determinar el estimado de los coeficientes genéticos P1 y P5, primero se determina la duración de las etapas fenológicas, según definidos en el Cuadro 1. Se observó que tanto el híbrido Pioneer como 'Mayorbela' germinaron a seis días después de la siembra (DDS), seguidos por 'Chulo' y 'Diente de Caballo' con 7 y 8 DDS, respectivamente. La germinación fue medianamente tardía, considerando que las plantas no sufrieron estrés hídrico. En el 2005 el híbrido Pioneer germinó 4 DDS, mientras que 'Mayorbela' germinó a los 5 DDS. 'Diente de Caballo' y ‘Chulo' germinaron a los 8 y 6 DDS, respectivamente. La germinación ocurrió en el intervalo esperado de tiempo (Cuadro 2).

En el 2004 la diferenciación de la yema apical se observó a los 24, 26, 27, y 31 DDS, para el híbrido Pioneer, 'Mayorbela', 'Diente de Caballo' y 'Chulo', respectivamente. Cabe señalar que en el 2005 los genotipos tardaron de uno a cuatro días adicionales para que se diferenciara la yema apical, excepto 'Mayorbela' que tardó seis días adicionales.

La aparición de la inflorescencia masculina ocurrió a los 51, 56, 61, y 59 DDS, para el híbrido Pioneer y las variedades 'Mayorbela', 'Diente de Caballo' y 'Chulo', respectivamente. En el 2005, la inflorescencia masculina apareció más temprano en todos los genotipos: 49, 54, 52 y 52 DDS, para los genotipos híbrido Pioneer, 'Mayorbela', 'Diente de Caballo' y 'Chulo', respectivamente. En el caso de 'Diente de Caballo' apareció nueve días antes que en el 2004.

La aparición de la inflorescencia femenina en el 2004 ocurrió a los 56, 58, 63 y 61 DDS para los genotipos híbrido Pioneer, 'Mayorbela', 'Diente de Caballo' y 'Chulo', respectivamente. En el 2005, la aparición de la inflorescencia femenina ocurrió entre uno y ocho días antes que el año anterior, presentando 'Mayorbela' solo un día de diferencia (Cuadro 2).

Para el experimento realizado en el 2004, la fecha de madurez fisiológica ocurrió a los 93, 98, 100 y 83 DDS, para los genotipos híbrido Pioneer, 'Mayorbela', 'Diente de Caballo'y 'Chulo', respectivamente. En el caso del 2005 este evento fenológico tardó de tres a cinco días menos que en el 2004 en todos los genotipos, excepto en el maíz 'Chulo' que tardó 11 días adicionales (90, 95, 95 y 94 DDS con los genotipos en el mismo orden mencionado para el 2004) (Cuadro 2).

Con esta información se determinaron los coeficientes genéticos P1 y P5 para todos los genotipos. En el 2004 se obtuvieron valores bajos de P1 (entre 219 y 253 Td para 'Mayorbela', 'Diente de Caballo' y el híbrido de Pioneer), excepto para 'Chulo', que se mantuvo dentro del valor 
CUaDRo 2.-Duración de las etapas fenológicas (DDS) de los genotipos de maíz sembrados durante 2004 y 2005.

\begin{tabular}{|c|c|c|c|c|c|c|c|c|c|c|}
\hline \multirow[t]{2}{*}{ Genotipo } & \multicolumn{2}{|c|}{ Emergencia } & \multicolumn{2}{|c|}{$\begin{array}{l}\text { Diferenciación } \\
\text { Yema Apical }\end{array}$} & \multicolumn{2}{|c|}{$\begin{array}{c}\text { Inflorescencia } \\
\text { Masculina }\end{array}$} & \multicolumn{2}{|c|}{$\begin{array}{c}\text { Inflorescencia } \\
\text { Femenina }\end{array}$} & \multicolumn{2}{|c|}{$\begin{array}{c}\text { Madurez } \\
\text { Fisiológica }\end{array}$} \\
\hline & 2004 & 2005 & 2004 & 2005 & 2004 & 2005 & 2004 & 2005 & 2004 & 2005 \\
\hline $\begin{array}{l}\text { 'Mayorbela' } \\
\text { Híbrido Pioneer }\end{array}$ & $\begin{array}{l}6 \\
6\end{array}$ & $\begin{array}{l}5 \\
4\end{array}$ & $\begin{array}{l}26 \\
24\end{array}$ & $\begin{array}{l}32 \\
28\end{array}$ & $\begin{array}{l}56 \\
51\end{array}$ & $\begin{array}{l}54 \\
49\end{array}$ & $\begin{array}{l}58 \\
56\end{array}$ & $\begin{array}{l}57 \\
52\end{array}$ & $\begin{array}{l}98 \\
93\end{array}$ & $\begin{array}{l}95 \\
90\end{array}$ \\
\hline 'Diente de Caballo' & 8 & 8 & 27 & 31 & 61 & 52 & 63 & 55 & 100 & 95 \\
\hline 'Chulo' & 7 & 6 & 31 & 32 & 59 & 52 & 61 & 55 & 83 & 94 \\
\hline
\end{tabular}

${ }^{1}$ DDS - días después de la siembra 
esperado con $362 \mathrm{Td}$ (Cuadro 3). En el experimento del 2005, los valores de P1 fueron más altos en comparación con los obtenidos en el 2004 y en otros estimados publicados para cultivares tropicales (Gungula et al., 2003). Los valores fueron 422, 352, 479 y 405 Td para 'Mayorbela', híbrido Pioneer, 'Diente de Caballo' y 'Chulo', respectivamente (Cuadro 3).

En el 2004, el coeficiente genético P5 de todos los genotipos se mantuvo cerca de los valores reportados para otros cultivares (Gungula et al., 2003), observándose entre 691 y 757 Td, excepto para maíz 'Chulo', que obtuvo un valor de $413 \mathrm{Td}$. En el 2005, los valores de P5 se mantuvieron dentro de los valores esperados (Ritchie et al., 1986): 755, 756, 795 y 777 para 'Mayorbela', híbrido Pioneer, 'Diente de Caballo’ y 'Chulo', respectivamente (Cuadro 3 ).

Coeficientes $G 2$ y $G 3$

En el 2004 el híbrido Pioneer obtuvo el valor mayor de G2 con 616 granos por mazorca, seguido por 'Mayorbela' con 566 granos/mazorca, 'Diente de Caballo' con 533 granos por mazorca y 'Chulo' con 529 granos/mazorca. El maíz 'Chulo' se resembró cuando ya los otros genotipos estaban establecidos, por lo que se mantuvo más pequeño durante su desarrollo, siendo el estimado de G3 el más bajo. Sin embargo, el maíz ‘Chulo’ alcanzó un G2 similar al de los otros cultivares (Cuadro 4). En el 2005, el estimado de G2 fue de 590, 561 y 558 granos por mazorca, para el híbrido Pioneer y los cultivares 'Chulo' y 'Mayorbela', respectivamente. El análisis de varianza indicó que no había diferencia significativa entre los cultivares para el número de granos por mazorca (análisis no incluido). El G2 se mantuvo dentro de los valores esperados determinando un buen estimado para el número de granos por mazorca para 'Mayorbela', 'Diente de Caballo', 'Chulo', y el híbrido Pioneer en ambos años de siembra y bajo las condiciones de clima en Lajas.

Se realizó un análisis de regresión lineal entre la acumulación de biomasa de la semilla y el tiempo transcurrido desde la fecha de

Cuadro 3.-Estimados de coeficientes genéticos de los genotipos de maíz sembrados en Lajas.

\begin{tabular}{lccccc}
\hline & \multicolumn{3}{c}{$\mathrm{P} 1^{1}$} & & \multicolumn{2}{c}{$\mathrm{P}^{2}$} \\
\cline { 2 - 3 } \cline { 5 - 6 } Genotipo & 2004 & 2005 & & 2004 & 2005 \\
\hline Mayorbela' & 253 & 422 & & 757 & 755 \\
Híbrido Pioneer & 219 & 352 & & 715 & 756 \\
'Diente de Caballo' & 252 & 479 & & 691 & 795 \\
'Chulo' & 362 & 405 & & 413 & 777 \\
\hline
\end{tabular}

${ }^{1} \mathrm{P} 1$ - Periodo, en Td, desde emergencia de la semilla hasta el final de la etapa juvenil.

${ }^{2} \mathrm{P} 5$ - Período, en Td, entre la fecha de florecida hasta fecha de madurez fisiológica. 
Cuadro 4.-Estimados de los coeficientes $G 2$ y G3 de los genotipos de maiz sembrados en Lajas.

\begin{tabular}{lccccc}
\hline & \multicolumn{3}{c}{$\mathrm{G}^{1}{ }^{1}$} & & \multicolumn{3}{c}{$\mathrm{G}^{2}$} \\
\cline { 2 - 3 } \cline { 5 - 6 } Genotipo & 2004 & 2005 & & 2004 & 2005 \\
\hline 'Mayorbela' & 566 & 558 & & 6.7 & 6.9 \\
Híbrido Pioneer & 616 & 590 & & 7.7 & 8.5 \\
'Diente de Caballo' & 533 & - & & 5.7 & 9.6 \\
'Chulo' & 529 & 561 & & 3.7 & 7.1 \\
\hline
\end{tabular}

${ }^{1} \mathrm{G} 2$ - Número potencial de granos por mazorca.

${ }^{2} \mathrm{G} 3$ - Velocidad de llenado del grano durante la etapa lineal bajo óptimas condiciones ( $\mathrm{mg} /$ día).

la aparición de la inflorescencia femenina (DDF) hasta la fecha de madurez fisiológica para estimar el coeficiente genético G3 con datos del 2004 y 2005 (Figuras 1 y 2). Este estimado se obtiene del valor de la pendiente de la regresión lineal. Por definición, en el análisis de regresión no se incluye la fase lenta de acumulación de biomasa (<10 DDF) ni la fase decreciente ( $>35 \mathrm{DDF}$ ) por ser no-lineales (Figura 1) Se observó que la mayor acumulación la obtuvo el híbrido Pioneer con $7.7 \mathrm{mg} /$ día, luego 'Mayorbela' con $6.7 \mathrm{mg} /$ día, seguido por 'Diente de Caballo' con $5.7 \mathrm{mg} /$ día, y por último y con una muy baja acumulación de materia seca 'Chulo' con $3.7 \mathrm{mg} /$ día (Cuadro 4). Los $\mathrm{R}^{2}$ muestran una regresión lineal confiable con valores de 0.92 ,
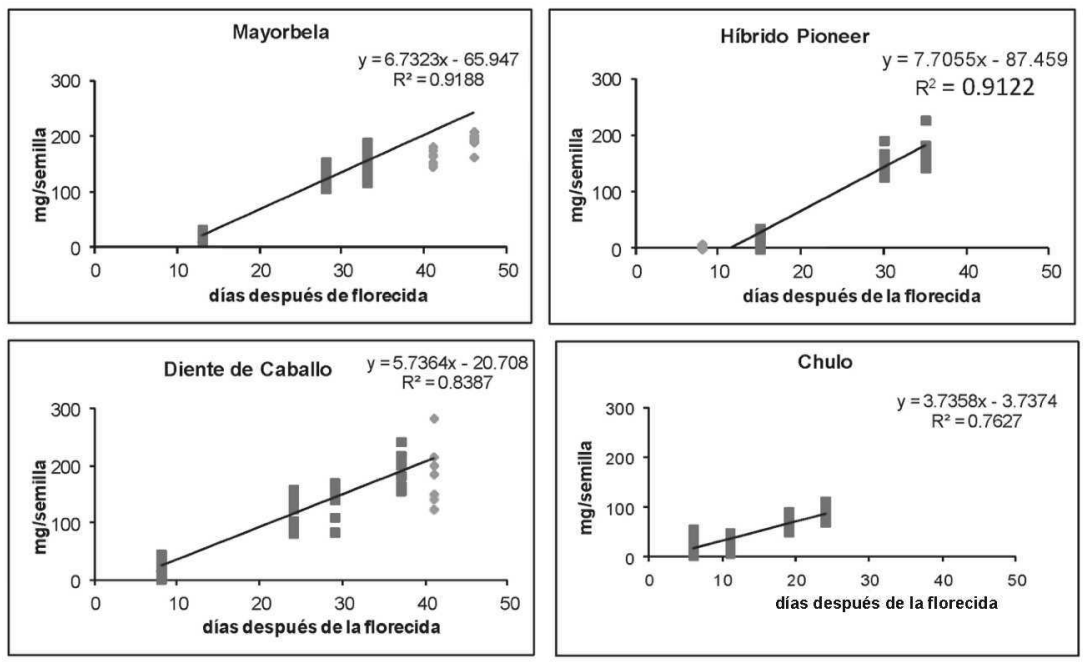

Figura 1. Análisis de regresión entre días después de la fecha de florecida y la biomasa acumulada en la semilla hasta madurez fisiológica en el 2004. El análisis de regresión no incluye la fase inicial ni la fase decreciente (puntos de color claro) de acumulación de biomasa en la semilla. La pendiente de la recta es un estimado de G3. 

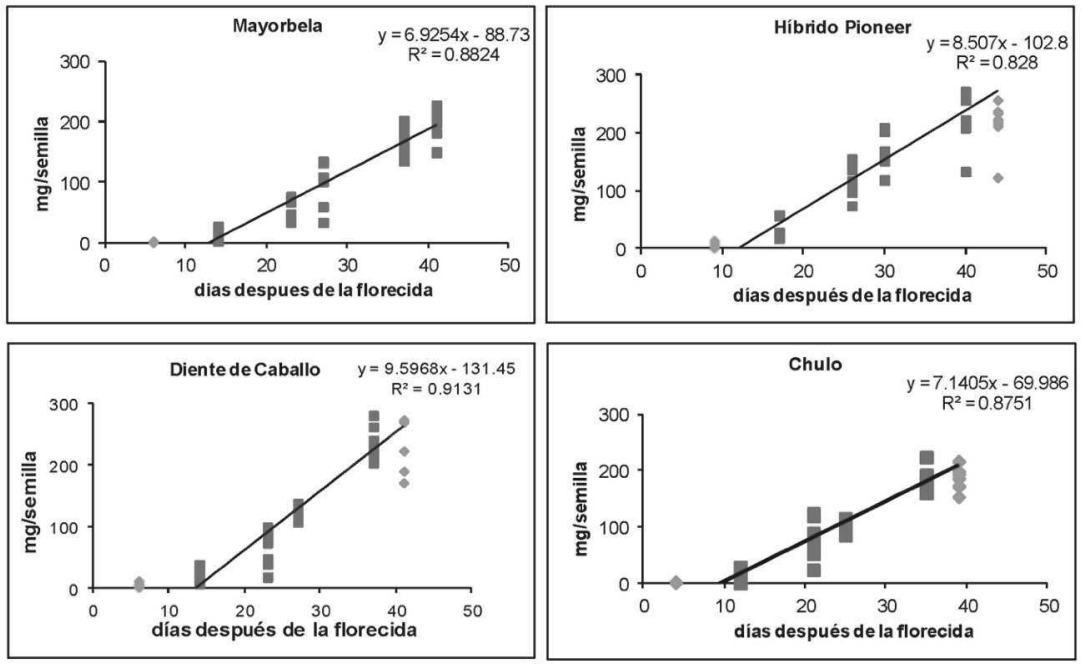

Figura 2. Análisis de regresión entre días después de la fecha de florecida y la biomasa acumulada en la semilla hasta madurez fisiológica en el 2005. El análisis de regresión no incluye la fase inicial ni la fase decreciente (puntos de color claro) de acumulación de biomasa en la semilla. La pendiente de la recta es un estimado de G3.

0.91, 0.84 y 0.76 para 'Mayorbela', el híbrido Pioneer, 'Diente de Caballo' y 'Chulo', respectivamente (Figura 1). Todos los genotipos habían completado la fase lineal de acumulación de materia seca en el grano entre 32 a 38 DDF, excepto 'Chulo' que lo completó a los 25 DDF.

En el 2005 el maíz 'Diente de Caballo' acumuló la mayor cantidad de materia seca en el grano, con $9.6 \mathrm{mg} /$ día, seguido por el híbrido Pioneer con $8.5 \mathrm{mg} /$ día. La acumulación de materia seca en 'Chulo' fue menor, con $7.1 \mathrm{mg} /$ día, seguido por 'Mayorbela', con $6.9 \mathrm{mg} /$ día. Los valores de $\mathrm{R}^{2}$ variaron entre 0.83 y 0.91 , mostrando una regresion lineal confiable para todos los genotipos, aunque menores a los obtenidos en el 2004 (Figura 2). Antes de los cuarenta días después de la florecida, todos los genotipos habían completado la fase lineal de acumulación de materia seca en el grano. 'Chulo'y 'Diente de Caballo' obtuvieron un G3 mayor en el año 2005. Este resultado se atribuye a que durante ese año el maíz 'Chulo' no tuvo problemas de germinación y su desarrollo estuvo a la par con el de los otros genotipos. Por otra parte, el maíz 'Diente de Caballo' tuvo baja germinación afectándose la densidad de siembra y permitiéndole a la planta mayor espacio y mejor intercepción de luz, lo que contribuye a mayor acumulación de materia seca en el grano. 
Índice de área foliar, acumulación de biomasa y rendimiento

Los datos sobre crecimiento, expresado por acumulación de biomasa e índice de área foliar (IAF), se presentan en el Cuadro 5. No se detectaron diferencias significativas para la interacción ni efecto principal para biomasa total para los genotipos evaluados. En un estudio similar realizado en el mismo suelo en el 2001, el maíz dulce 'Suresweet' sembrado a una densidad de 6.4 plantas por metro cuadrado produjo una biomasa total de $20.5 \mathrm{~g} / \mathrm{planta}$ a los 43 DDS (Román-Paoli y Sotoma-

CuADRo 5.-Acumulación de biomasa ( $\mathrm{g} /$ planta) e indice de área foliar (IAF) de los genotipos de maiz sembrados en Lajas.

\begin{tabular}{|c|c|c|c|c|c|c|}
\hline \multirow[b]{2}{*}{ Genotipo } & \multicolumn{3}{|c|}{2004} & \multicolumn{3}{|c|}{$2005^{2}$} \\
\hline & $\mathrm{DDS}^{1}$ & Biomasa & $\mathrm{IAF}$ & DDS & Biomasa & IAF \\
\hline 'Mayorbela' & $\begin{array}{l}13 \\
27 \\
41 \\
54 \\
79 \\
94\end{array}$ & $\begin{array}{r}0.42 \\
5.59 \\
41.90 \\
101.72 \\
183.30 \\
202.54\end{array}$ & $\begin{array}{l}0.024 \\
0.285 \\
1.562 \\
2.088 \\
2.244 \\
1.930\end{array}$ & $\begin{array}{r}19 \\
33 \\
48 \\
61 \\
78 \\
95 \\
102\end{array}$ & $\begin{array}{r}1.09 \\
8.06 \\
25.00 \\
73.83 \\
182.33 \\
212.68 \\
269.60\end{array}$ & $\begin{array}{l}0.21 \\
0.70 \\
1.33 \\
1.31 \\
1.01 \\
- \\
-\end{array}$ \\
\hline Híbrido Pioneer & $\begin{array}{l}13 \\
27 \\
41 \\
54 \\
79 \\
94\end{array}$ & $\begin{array}{r}0.48 \\
16.52 \\
32.29 \\
111.14 \\
156.31 \\
227.69\end{array}$ & $\begin{array}{l}0.027 \\
0.347 \\
1.676 \\
2.013 \\
1.949 \\
1.554\end{array}$ & $\begin{array}{r}19 \\
33 \\
48 \\
61 \\
78 \\
95 \\
102\end{array}$ & $\begin{array}{r}0.90 \\
17.3 \\
23.8 \\
70.3 \\
171.3 \\
166.9 \\
185.2\end{array}$ & $\begin{array}{l}0.34 \\
0.89 \\
0.97 \\
1.04 \\
0.80 \\
- \\
-\end{array}$ \\
\hline 'Diente de Caballo' & $\begin{array}{l}13 \\
27 \\
41 \\
54 \\
79 \\
94\end{array}$ & $\begin{array}{r}0.41 \\
3.03 \\
28.64 \\
113.37 \\
147.44 \\
199.51\end{array}$ & $\begin{array}{l}0.017 \\
0.197 \\
1.461 \\
1.886 \\
1.796 \\
2.111\end{array}$ & $\begin{array}{r}19 \\
33 \\
48 \\
61 \\
78 \\
95 \\
102\end{array}$ & $\begin{array}{l}\bar{z}^{3} \\
- \\
- \\
-\end{array}$ & $\begin{array}{l}z \\
z \\
z \\
-\end{array}$ \\
\hline 'Chulo' & $\begin{array}{l}13 \\
27 \\
41 \\
54 \\
79 \\
94\end{array}$ & $\begin{array}{l}- \\
- \\
- \\
-\end{array}$ & $\begin{array}{l}- \\
- \\
- \\
-\end{array}$ & $\begin{array}{r}19 \\
33 \\
48 \\
61 \\
78 \\
95 \\
102\end{array}$ & $\begin{array}{r}0.77 \\
11.87 \\
33.75 \\
64.43 \\
149.14 \\
247.96 \\
231.50\end{array}$ & $\begin{array}{l}0.21 \\
0.81 \\
1.20 \\
1.13 \\
1.08 \\
- \\
-\end{array}$ \\
\hline Análisis de Varianza & & \multicolumn{5}{|c|}{ Probabilidad } \\
\hline $\begin{array}{l}\text { bloque } \\
\text { variedad } \\
\text { bloque } \times \text { variedad } \\
\text { tiempo } \\
\text { tiempo } \times \text { variedad }\end{array}$ & & $\begin{array}{l}0.2944 \\
0.3036 \\
0.4087 \\
0.0001 \\
0.4734\end{array}$ & $\begin{array}{l}0.9011 \\
0.5085 \\
0.0113 \\
0.0001 \\
0.0034\end{array}$ & & $\begin{array}{l}0.2955 \\
0.9280 \\
0.0001 \\
0.0625\end{array}$ & $\begin{array}{l}0.8505 \\
0.3908 \\
0.0001 \\
0.0052\end{array}$ \\
\hline
\end{tabular}

${ }^{1} \mathrm{DDS}$ - días después de la siembra.

${ }^{2}$ En el 2005 el diseño experimental fue completamente aleatorizado.

${ }^{3}$ No se determinó área foliar ni biomasa debido a una pobre germinación. 
yor-Ramírez, 2004), cantidad menor que la producida por cualquiera de los genotipos evaluados. En el caso de IAF existe una interacción significativa entre tiempo y genotipo para ambos años del estudio. En ambos años el híbrido Pioneer poseía un IAF mayor que los demás genotipos hasta aproximadamente 40 DDS; luego 'Mayorbela' incrementó su IAF hasta 2.2, lo cual resultó superior en un $20 \%$.

Se determinó el número total de hojas para los diferentes genotipos. 'Diente de Caballo' produjo el mayor número total de hojas con 25, mientras que 'Mayorbela' produjo 24, y el híbrido Pioneer, 23. No se pudo obtener el número total de hojas para 'Chulo'.

En el 2005, 'Mayorbela' produjo el mayor número de hojas, con 19, seguido por 'Diente de Caballo' y 'Chulo' con 18 hojas. El menor número de hojas se obtuvo con el híbrido Pioneer, con un total de 16. Una planta de maíz en los trópicos puede producir hasta 30 hojas (Paliwal y Granados, 2001).

En este experimento el maíz 'Chulo' completó su ciclo de vida en solo 83 días. Se ha reportado que este cultivar tiene un ciclo de cultivo entre 105 y 110 días (Meneses-Ojeda, 1992). El maíz 'Chulo' produjo 2,176 $\mathrm{kg} / \mathrm{ha}$; un rendimiento bajo en comparación con los otros genotipos, posiblemente debido a una mayor competencia por agua y luz con los demás genotipos. En el 2004 'Mayorbela' tuvo un rendimiento de 4,148 $\mathrm{kg} / \mathrm{ha}$, seguido por el híbrido Pioneer con un rendimiento de $3,986 \mathrm{~kg} /$ ha, pero no se detectó diferencia significativa entre estos. 'Diente de Caballo' tuvo un rendimiento de 3,396 kg/ha (Cuadro 6). El híbrido Pioneer se afectó con una enfermedad preliminarmente identificada como roya, entre 60 y 65 DDS durante el 2004, lo que pudo haber reducido su rendimiento. Tenemos que recalcar que los rendimientos fueron bajos ya que en este tipo de estudio la planta debe expresar su potencial individual, por lo que deliberadamente se siembran dichos genotipos a una densidad poblacional baja (3.6 plantas $\left./ \mathrm{m}^{2}\right)$.

CuAnRo 6.-Rendimiento ( $\mathrm{kg} / \mathrm{ha}$ ) de los genotipos de maiz sembrados en Lajas.

\begin{tabular}{llc}
\hline Genotipo & 2004 & $2005^{1}$ \\
\hline Mayorbela' & $4,148 \mathrm{a}$ & $3,313 \mathrm{~b}$ \\
Híbrido Pioneer & $3,986 \mathrm{ab}$ & $3,845 \mathrm{a}$ \\
'Diente de Caballo' & $3,396 \mathrm{~b}$ & - \\
'Chulo' & $2,176 \mathrm{c}$ & $3,098 \mathrm{~b}$
\end{tabular}

Análisis de varianza

Probabilidad

\begin{tabular}{llc}
\hline Bloque & 0.0226 & $0 . \overline{0} 11$ \\
Variedad & 0.0004 & 0.0211 \\
\hline
\end{tabular}

\footnotetext{
${ }^{1}$ En el año 2005 el diseño experimental fue uno completamente aleatorizado; en el análisis de varianza el número de plantas por parcela se utilizó como covariable.
} 
En el 2005 el híbrido Pioneer tuvo el rendimiento más alto, con $3,845 \mathrm{~kg} / \mathrm{ha}$, seguido por 'Mayorbela', con 3,313 kg/ha (Cuadro 6). El rendimiento obtenido en 2005 fue menor para 'Mayorbela' y el híbrido Pioneer en comparación al del año 2004, sin embargo, el redimiento de 'Chulo' fue mayor en 2005 que en 2004.

Esta es la primera vez que se realiza un estudio detallado del crecimiento y desarrollo de los cultivares locales de maíz y la primera vez que estos son comparados con un híbrido comercial que en principio tiene un potencial de rendimiento mayor. Estos cultivares de polinización abierta han sido evaluados y mejorados individualmente a través de los años bajo diferentes prácticas de manejo. En este estudio 'Mayorbela' se comportó a la par o superó al híbrido transgénico comercial en los parámentros medidos. El número de hojas y el IAF del híbrido Pioneer fueron menores a los producidos por 'Mayorbela', sin embargo, el híbrido Pioneer mostró igual o mayor productividad. Este resultado se puede deber a varias razones, entre las cuales podrían estar que el híbrido Pioneer es más eficiente en la utilización de la radiación solar debido a una estructura vertical del dosel foliar que permite mayor absorción. Otra razón podría ser que es un híbrido transgénico para tolerancia a Lepidópteros.

El potencial de rendimiento de 'Chulo' es muy bajo por lo que no se recomienda su siembra a menos que sea para uso específico de alimentación de gallos de pelea, por su semilla de tamaño pequeño. Los estimados de los CG publicados son de cultivares adaptados a condiciones tropicales bajo clima tropical. Estos estimados obtenidos en el campo sirven de base para utilizar el modelo CERES-Maize como una herramienta para el manejo de maíz a gran escala bajo condiciones de Puerto Rico.

\section{LITERATURA CITADA}

Beaver, J., M. Saliceti y J. Sud, 2006. Release of maize [Zea mays (L.) Walp.] open pollinated cultivar Mayorbela 05. J. Agric. Univ. P.R. 90:253-257.

Carberry, P. S., R. C. Muchow y R. L. McCown, 1989. Testing the CERES-Maize simulation model in semi-arid tropical environment. Field Crops Res., 20:297-315.

Carte, 1965. Soil Survey of Lajas Valley Area of South P.R. United State Department of Agriculture, Soil Conservation Services; in cooperation with the University of Puerto Rico; College of Agricultural Sciences. Washington DC. pp. 93-96 \&110112.

Duncan, W. G. y A. L. Hatfield, 1964. A method for measuring the daily growth of corn kernels. Crop Science 63:10-16.

DSSAT. (The Decision Support System for Agrotechnology Transfer and International Benchmark Project Sites Network for Agrotechnology), 1998. Página de la información de DSSAT, IBSNAT, http://www.icasanet.org/dssat/index.html 
Freebaim, D., M. Littleboy y G. Wockner, 1993. Understanding simulation models - a tool to study cropping systems. Agricultural Production System series: Number 1. CSIRO Australia. pp. 2.

Gierbolini, E. R., 1975. Soil Survey of Mayagüez Area of Western P.R. United States Department of Agriculture, Soil Conservation Services; in cooperation with the University of Puerto Rico; College of Agricultural Sciences. Washington DC. pp. 2-45.

Gungula, D. T., J. G. Kling y A. O. Togun, 2003. CERES-Maize Prediction of maize phenology under nitrogen-stressed conditions in Nigeria. Agron. J. 95: 892-899.

Hunt, L. A., S. Parajasingham, J. W. Jones, G. Hoogenboom, D. Imamura y R. M. Ogoshi, 1993. Gencalc: Software to facilitate the use of crop models for analyzing field experiments. Agron. J. 85: 1090-1094.

IBSNAT. International Benchmark Project Sites Network for Agrotechnology Transfer, 1993. The IBSNAT decade. Department of Agronomy and Soil Science, College of Tropical Agriculture and Human Resources, University of Hawaii, Honolulu, HI.

Jones, C. A. y J. R. Kiniry, 1986. CERES-Maize: A simulation model of maize growth and development, Texas A\&M Univ. Press, College Station, pp. 113-144.

Kiniry, J. R., J. T. Ritchie, R. L. Musser, E. P. Flint y W. C. Iwig, 1983. The Photoperiod Sensitive Interval in Maize. Agron. J. 75: 686-691.

López, R., 1994. Progreso de selección recurrente recíproca en cuatro poblaciones de maíz y su comportamiento agronómico en relación al gusano de la mazorca y el cogollero (Lepidóptero: Noctuidae). Tesis de Maestría. Universidad de Puerto Rico en Mayagüez. pp.9-20.

Lugo López, M. A., 1995. El Valle de Lajas: 50 años después. Acta Cientifica 9: 57-69.

Meneses-Ojeda, A., 1992. Aceptabilidad y efecto de niveles de nitrógeno y densidades poblacionales en la variedad de maíz, Zea mays, Chulo. Tesis de Maestría. Universidad de Puerto Rico en Mayagüez. pp.33-82.

Nielsen, R. L., P. R. Thomison, G. A. Brown, A. L. Halter, J. Wells y K. L. Wuethrich, 2002. Delayed planting effects on flowering and grain maturation of dent corn. Agron. J. 94:549-558.

Ogoshi, R. M., L. A. Hunt, J. W. Jones y G. Y. Tsuji, 1989. Determination and application of field derived genetic coefficients for CERES-Maize crop model. Annual Meetings. American Society of Agronomy. Crop Science Society of America. Las Vegas, Nevada. October 15-20, 1989. Agronomy Abs. pp. 20.

Paliwal, L. y G. Granados, 2001. El maíz en los Trópicos: Mejoramiento y Producción. Organización de las Naciones Unidas para la Agricultura y la Alimentación. pp. 376.

Ritchie, S. W., J. J. Hanway y G. O. Benson, 1992. How a corn plant develops. Special Report, No.48. Iowa State University of Science and Technology Cooperative Extension Service Ames, Iowa. Reprinted June 1993. pp. 11-32.

Ritchie, J. T., J. R. Kiniry, C. A. Jones y P. T. Dyke, 1986. Model Inputs. CERES-Maize: A Simulation Model of Maize Growth and Development. Texas A \& M University Press, College Station, pp. 113-144.

Román-Paoli, E. y D. Sotomayor-Ramírez, 2004. Soil conditioner efficacy on Lajas Valley sweet corn production. J. Agric. Univ. P.R. 88:97-108.

Román-Paoli, E., S. Welch y R. Vanderlip, 2000. Comparing genetic coefficients estimation methods using the CERES-Maize model. Agric. Sys. 65:29-47.

Sotomayor Ríos, A., 1979. Performance of twelve corn hybrids and selections in three consecutive crops on three same sites in the same year. J. Agric. Univ. P.R. 63:170179.

Sotomayor Ríos, A., C. Torres y M. Ellis, 1980. Effect of plant density on yield and plant characteristics of twelve corn hybrids and selections. J. Agric. Univ. P.R. 64:407413. 
Tsuji G. Y., G. Uehara y S. Balas, 1994. Decision Support System for Agrotechnology Transfer. Version 3.0. Crop simulation software. Univ. of Hawaii, Honolulu. Annual Meetings. American Society of Agronomy. Crop Science Society of America. Seattle, Washington. November 13-18, 1994. Agronomy Abs. pp. 99.

Tsuji, G. Y., G. Hogenboom y P. K. Thornton (ED), 1998. Understanding Options for Agricultural Production. Kluwer Academic. 